\title{
Object-based attention with endogenous cuing and positional certainty
}

\author{
ZHE CHEN \\ University of Canterbury, Christchurch, New Zealand \\ AND \\ KYLE R. CAVE \\ University of Massachusetts, Amherst, Massachusetts
}

\begin{abstract}
Previous studies have concluded that object-based attention does not always arise if attention is cued endogenously (Macquistan, 1997) or if the target location is known with certainty (Shomstein \& Yantis, 2002). In the Experiments reported here, we found object-based attention even when the locations of the two targets were known with certainty due to presentation of an endogenous cue. However, object-based attention can be prevented by limiting the exposure time of the object stimuli. These findings provide additional evidence against a search prioritization account of object-based attention. They lead to a fuller view of the role of object boundaries in directing attention. Object-based attention is frequently used spontaneously, even when the object boundaries are irrelevant to the task. However, object segmentation is not necessary for all tasks, because attention can be allocated before the stimulus has been segregated into objects.
\end{abstract}

When attention is cued to a specific location in space, the attentional facilitation can be greater for locations near the cued location than for those farther away and can also be greater for locations that are within the same object boundaries as the cued location is (Egly, Driver, \& Rafal, 1994; Moore, Yantis, \& Vaughan, 1998), showing that attention can select locations, but also has a tendency to select an entire object. Both location-based attention and object-based attention have been demonstrated in a variety of different visual tasks. The nature of object-based attention is a particularly important question in visual cognition, because it reflects the relationships between early visual processing, higher level object representations, and response selection. This study will focus on the factors that govern object-based attention.

Object-based attention has been found in many conditions. It arises when different components of a stimulus display are perceived as part of a single object or of a single perceptual group (Duncan, 1984; Harms \& Bundesen, 1983; Kim \& Cave, 2001; Kramer \& Jacobson, 1991), when a target can occur at one of several possible locations (Chen, 1998; Egly et al., 1994; Moore et al., 1998), and when the targets are either on the same object or on different objects (Kramer \& Watson, 1995; Lavie \& Driver, 1996).

\section{Exogenous Versus Endogenous Cuing}

Although object effects have been found in a variety of paradigms, object boundaries do not always influence the allocation of attention (Arrington \& Dagenbach, 2000; Macquistan, 1997; Neely \& Dagenbach, 1996). For instance, Macquistan found no object effects when attention was cued to a location endogenously rather than exogenously. The type of cuing does not solely determine the nature of attention, however, because Goldsmith and Yeari (2003) noted that the differential results associated with the exogenous and endogenous cues depend on the extent of attentional focus on the two types of trials. Because exogenous cues are typically situated peripherally, whereas endogenous cues are located centrally, a broad attentional focus would be more likely to be used with exogenous cues, just as a narrow attentional focus would be more likely to be used with endogenous cues. In Goldsmith and Yeari's experiments, object-based attention arose only when attention was distributed widely over a large area and not when it was narrowly focused (see also Abrams \& Law, 2000, for object effects with endogenous cuing).

\section{Positional Uncertainty}

Object-based attention has often been explained with a sensory enhancement account that stresses the improved quality of an early sensory representation of the selected item or its region (Desimone \& Duncan, 1995). Shomstein and Yantis (2002) questioned this account, however, because object-based attention is less likely to occur when the target location is known in advance. In their experiments, participants identified a target letter in the pres-

Z. Chen, zhe.chen@canterbury.ac.nz 
ence of distractor letters. The target and distractor letters appeared within the boundaries of three rectangles. On some trials, the target and distractor letters were all within the same object, whereas on other trials, the target letter and the distractors were within different objects, so that there were object boundaries between the target and the distractors. When the location of the target letter was not known in advance, so that attention had to be spread widely across the display before the letters appeared, the interference from the distractor letters was less when they were on different objects from the target letter: The object boundaries limited the interference between distractor and target. However, when the target always appeared at the same location in the center of the display, so that attention could be narrowly focused at this location before the letters appeared, the distractor inhibition was no longer affected by object boundaries. Given that object-based attention arose when search was necessary, but not when advance knowledge of the target location made search unnecessary, Shomstein and Yantis rejected the sensory enhancement account in favor of an alternative attentional prioritization account that attributes the attentional facilitation to the higher priority that the selected item or its region receives during search.

However, the prioritization account conflicted with results from Experiments by Chen and Cave (2006), demonstrating that the effect of object boundaries on distractor interference could be reinstated if, on some trials, the display included only one or two of the three rectangles making up the full configuration. Presumably, these partial displays prompted participants to think of the display as consisting of separate objects, rather than as a single configuration, even when all the rectangles appeared together. Thus, even with full certainty about the target position, so that attention can be focused narrowly at the center, participants will still allocate their spatial attention to conform to the boundaries of the object surrounding the target if they are prompted to interpret the different parts of the display as separate objects. These results show that top-down interpretation of ambiguous configurations can facilitate the establishment of object representations, which, in turn, lead to object-based distribution of attention. Furthermore, these results suggest that object-based attention arises because of sensory enhancement of the selected item or its region.

\section{Display Duration}

Prior research has suggested that object-based attention is influenced by the quality of object representations (Avrahami, 1999). Given that the exposure duration of a stimulus pattern contributes to the formation of object representation, it should also modulate the manifestation of object effects. Previous findings are consistent with this view. Whereas object effects were frequently observed when the exposure duration of a stimulus was relatively long (Egly et al., 1994; Kramer \& Jacobson, 1991), they were less reliably elicited when it was short (e.g., Lavie \& Driver, 1996; Law \& Abrams, 2002; but see Duncan, 1984; Kramer \& Watson, 1995). Law and Abrams used a spatial cuing paradigm with a peripheral cue in several experiments, and they obtained object effects when the stimulus duration was $186 \mathrm{msec}$. However, when the exposure duration was decreased to $129 \mathrm{msec}$ in a subsequent experiment, no object effect was found. The latter result was similar to the finding of an earlier experiment by Lavie and Driver, who also failed to observe any object effect in a similar paradigm, with an exposure duration of $130 \mathrm{msec}$. These results show that object-based attention requires time to develop.

\section{What Turns Object-Based Attention On and Off?}

The picture that has emerged of attentional control is fairly complex. Object-based attention is more likely to appear with uncertainty about the target position that makes search necessary (Shomstein \& Yantis, 2002), but it also appears in some circumstances when search is unnecessary (Chen \& Cave, 2006). Object-based attention is more likely to be deployed when the extent of attentional focus is large (Goldsmith \& Yeari, 2003) but also occurs when attention can be narrowly focused, as long as the stimulus pattern is subjectively parsed as multiple objects (Chen, 1998; Chen \& Cave, 2006). Furthermore, the manifestation of the object effect has been linked to the exposure duration of the display (Law \& Abrams, 2002) and to the quality of the object representation within a stimulus pattern (Avrahami, 1999). These studies show that a number of factors influence the use of object-based attention, but the interactions between these factors that are necessary for object-based attention are not clear. Understanding these interactions will provide new clues as to when and how objects are segregated in visual processing.

One basic question involves the relationship between spatial cuing and knowledge of target location. Many of the Experiments exploring object-based attention have employed a cuing paradigm with positional uncertainty: The target has some probability of appearing at many different locations. Shomstein and Yantis's (2002) results suggest that positional uncertainty promotes object-based attention. Is the positional uncertainty necessary to produce object-based attention in Experiments with spatial cuing? Furthermore, when object-based attention does arise, does it require a certain amount of time for the object segregation to be established?

The Experiments presented here were designed to answer these questions. As in our previous study (Chen \& Cave, 2006), target locations were known before the targets appeared, and the stimulus patterns were made of three rectangles. However, unlike in our earlier experiments, the target locations were indicated by endogenous cues. Given the results from Macquistan (1997), one might predict that there would be no object-based attention in this task. However, there was an important difference in these new experiments. The task here was to make speeded same-different responses to two target letters that could appear either on the two ends of a single rectangle, or on two separate rectangles. Because two targets appeared simultaneously in different cued locations in all the conditions, participants had to adopt an attentional focus 
that was broad enough to include both targets in order to perform the task. We hypothesized that this widening of attention might make it more likely for object-based deployment of attention to occur. Although the locations of the targets varied from trial to trial, they were clearly indicated by an endogenous precue of $100 \%$ validity.

In Experiment 1, we examined the generality of our previous finding that object-based attention could be observed with invariant target location. In Experiment 2, we tested the hypothesis that the cue was not merely sensory in nature but was interpreted and used to guide attention. In Experiment 3, we investigated whether the object effect obtained in Experiment 1 required time to develop and was therefore subject to elimination when the exposure duration of the stimulus pattern was brief. In Experiments 4 and 5, we further confirmed that the cuing in these Experiments was indeed endogenous.

\section{EXPERIMENT 1}

The goal of Experiment 1 was to generalize the findings that Chen and Cave (2006) obtained with endogenous cuing. Participants saw stimulus displays that consisted of three rectangles (see Figure 1), followed by a central endogenous cue with $100 \%$ validity and two target letters at the ends of either the large rectangle or the two small rectangles. Because the cue was always valid, there was no need to search for the targets. Of particular interest was whether participants would still show an object effectthat is, faster responses to the letters when they were in the same rectangle rather than in two different rectangles.

\section{Method}

Participants. Fourteen undergraduate students from the University of Canterbury participated in the experiment in exchange for payment. All participants reported having normal or corrected-tonormal vision.
Apparatus and Stimuli. Stimulus displays were shown on the 13-in. RGB monitor of a Power Macintosh 6100/66 computer in a dimly lit room. The viewing distance between the participants and the monitor was approximately $60 \mathrm{~cm}$. MacProbe (Hunt, 1994) was used to display stimuli and to record responses.

All stimuli were presented against a homogeneous gray background. Each trial consisted of a fixation cross, a pattern of three rectangles that remained on the screen throughout a trial, a cue, and two target letters. The fixation cross was black, and the stimulus pattern was made of one large and two small blue-outlined rectangles. On half of the trials, the large rectangle was placed horizontally and the small rectangles vertically. On the rest of the trials, their orientation was reversed. At a viewing distance of $60 \mathrm{~cm}$, the fixation subtended $1.05^{\circ}$ of visual angle. The large rectangle subtended $12.04^{\circ} \times$ $1.72^{\circ}$, and the two small ones each subtended $5.06^{\circ} \times 1.72^{\circ}$. The small rectangles were situated on the sides of the large rectangle, with a gap of $0.1^{\circ}$ between the large rectangle and each of the small rectangles. The entire stimulus pattern was $12.04^{\circ}$ in both length and width. The cue was made of a white two-headed arrow, which subtended $1.15^{\circ}$ and was located at the center of the stimulus pattern. Its orientation was as likely to be vertical as horizontal. The letters were black uppercase Ts and Ls written in Geneva font with a point size of 30 . They were placed at two opposite ends of the stimulus pattern and were equally likely to be vertically or horizontally aligned. The center-to-center separation between the letters was $10.51^{\circ}$.

Design and Procedure. The experiment employed a withinparticipants design. The task was to indicate, as quickly and as accurately as possible, whether the target letters were the same (two Ts or two Ls) or different (one T and one L). The letters were equally likely to be the same or different. On half of the trials, they appeared on the same rectangle (the same-object condition), and on the rest of the trials, they appeared on different rectangles (the different-object condition).

Each trial started with the presentation of the fixation cross for $495 \mathrm{msec}$. After $510 \mathrm{msec}$ of blank screen, the stimulus pattern appeared. It stayed on the screen for $1,005 \mathrm{msec}$ before the cue was flashed for $120 \mathrm{msec}$. At the offset of the cue, two target letters were shown for $120 \mathrm{msec}$. The participants were instructed to make a speeded same-different response to the letters by using their index or middle fingers to press one of the two designated keys on the keyboard (the "." key if the letters were the same and the "/" key if the letters were different). To encourage them to perceive the stimulus

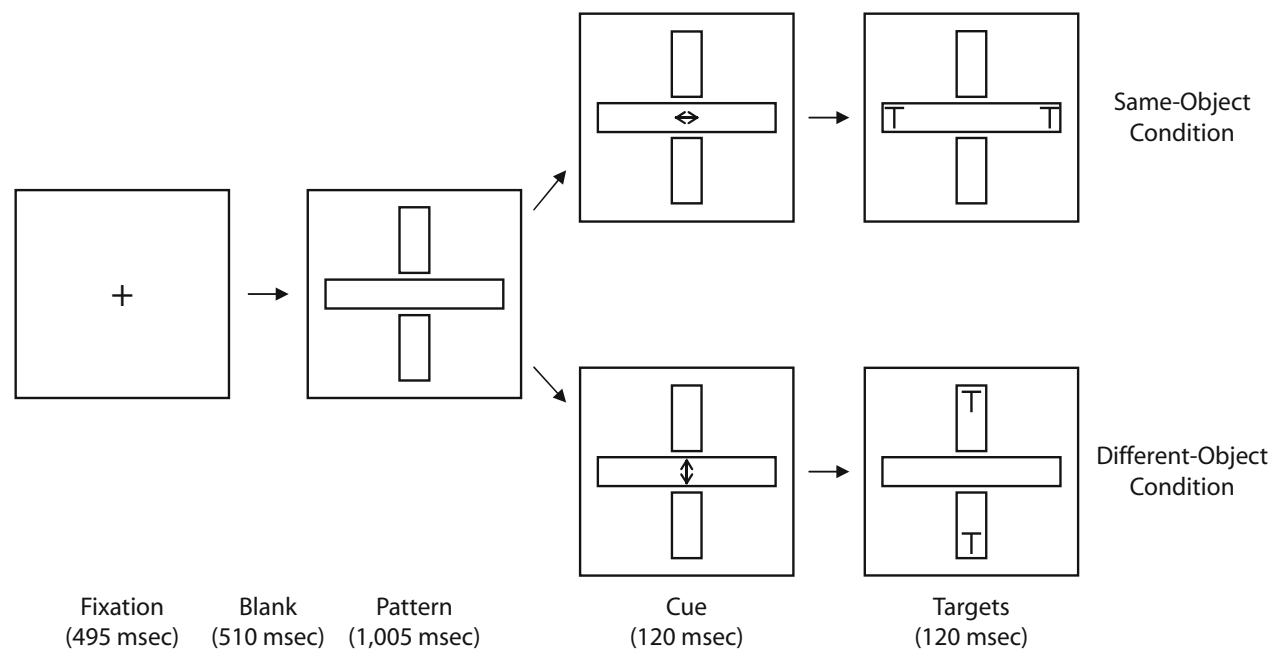

Figure 1. Examples of stimulus displays from Experiment 1. The task was to decide whether the letters were the same or different. The cue was $100 \%$ valid. 
Table 1

Mean Reaction Times (RTs, in Milliseconds) and Error Rates (Percentage Incorrect), With Standard Errors, for Experiment 1

\begin{tabular}{|c|c|c|c|c|}
\hline & \multicolumn{2}{|c|}{ RT } & \multicolumn{2}{|c|}{$\%$ Error } \\
\hline & $M$ & $S E$ & $M$ & $S E$ \\
\hline Same & 625 & 26.6 & 5.5 & 1.11 \\
\hline Different & 639 & 26.9 & 6.3 & 1.05 \\
\hline
\end{tabular}

pattern as being made of three distinct objects rather than one single configuration, the participants were shown the individual rectangles before they saw the combined form of the stimulus pattern. They then performed 32 practice trials, followed by four blocks of 80 trials with short breaks in between. The entire experiment took approximately $30 \mathrm{~min}$ to complete.

\section{Results and Discussion}

Table 1 shows the mean reaction times (RTs) and accuracy data. Two $t$ tests for dependent means were conducted, one on RTs and the other on error rates. RT was shorter when the target letters appeared on the same object ( $M=625 \mathrm{msec})$ than when they occurred on two different objects $(M=639 \mathrm{msec})[t(13)=3.99, p<.01]$. No significant effect was found for the accuracy data $[5.5 \%$ and $6.3 \%$ error rates for the same- and different-object conditions, respectively; $t(13)=1.10$, n.s.].

Consistent with prior research (Chen \& Cave, 2006), an object effect was found even though the locations of targets were known in advance because of a $100 \%$ valid endogenous cue. Because the cue appeared at the center, the participants presumably shifted attention from the cue to the target locations upon the onset of the target letters. However, despite the shift of attention, search was not necessary, since the target always occurred at the cued locations. This result provided converging evidence to our previous finding that object-based deployment of attention did not require visual search and could be obtained with foreknowledge of target locations. It is also consistent with Goldsmith and Yeari's (2003) finding that object-based attentional distribution is the result of the extent of spatial focus rather than the result of exogenous or endogenous cuing.

However, although the presence of a $100 \%$ valid cue made it unnecessary to search for the target, one may wonder whether the cue was interpreted or whether it was only sensory in nature. Because a neutral cue condition was not included in the experiment, we could not verify empirically that the participants indeed used the cue to guide attention. Experiment 2 was designed to address this issue. ${ }^{1}$

\section{EXPERIMENT 2}

Experiment 2 consisted of two types of trials: valid cue trials, which were identical to those in Experiment 1, and neutral cue trials, which had an uninformative cue instead of a valid one. The two types of trials were presented in separate blocks. If the cue was not interpreted in Experiment 1 , there was no reason to expect it to be utilized in Experiment 2. In other words, we would observe object effects regardless of whether the cue was valid or neutral.
However, if the cue in Experiment 1 helped guide attention, we should find different patterns of data across the two blocks in Experiment 2. Whereas participants would be motivated to shift attention from the cued location to the locations of the targets in the valid cue block, they would be less likely to do so in the neutral cue block because the cue did not provide information about the targets. There is evidence that shifting attention is conducive to object-based deployment of attention (Lamy \& Egeth, 2002; Shomstein \& Yantis, 2002).

\section{Method}

The method was similar to that of Experiment 1 except for the following differences. Each participant took part in two consecutive blocks. In the valid cue block, all aspects of a trial were identical to those in Experiment 1. In the neutral cue block, the cue was made of two double-headed arrows that formed the shape of a cross, and the participants were informed that the targets were equally likely to appear at the horizontal or vertical ends of the stimulus pattern. Each block consisted of 192 trials, with a short break in between. The order of the blocks was counterbalanced across the participants. Twenty new people from the same participant pool took part in the experiment.

\section{Results and Discussion}

The results are shown in Table 2. One participant's data were not included, because of high error rates that exceeded $45 \%$ in two conditions. A $2 \times 2$ repeated measures ANOVA on mean RTs revealed a significant main effect of object $[F(1,18)=14.88, p<.01]$ and an object $\times$ cue interaction $[F(1,18)=4.50, p<.05]$. No significant effect of cue was found $[F(1,18)<1$, n.s.]. These results suggest that RTs were faster when the target letters were on the same object $(M=613 \mathrm{msec})$ than when they were on different objects $(M=625 \mathrm{msec})$. Furthermore, the effect of object was larger when the cue was valid ( $M=$ $20 \mathrm{msec})$ than when it was neutral $(M=5 \mathrm{msec})$. Subsequent $t$ tests confirmed that whereas participants showed a significant object effect in the valid cue block $[t(18)=$ $3.93, p<.001]$, they did not do so in the neutral cue block $[t(18)=1.23$, n.s. $]$. No significant effects were found on the accuracy data $[F(1,18)=3.45$, n.s., for object; $F(1,18)<1$, n.s., for cue; and $F(1,18)=1.40$, n.s., for the object $X$ cue interaction].

The most important finding of Experiment 2 was the differential patterns of data on the valid and neutral cue trials. As expected, the result in the valid cue block replicated that of Experiment 1: Participants showed a sig-

Table 2

Mean Reaction Times (RTs, in Milliseconds) and Error Rates (Percentage Incorrect), With Standard Errors, for Experiment 2

\begin{tabular}{cccccc}
\hline & \multicolumn{2}{c}{ RT } & & \multicolumn{2}{c}{$\%$ Error } \\
\cline { 2 - 3 } \cline { 5 - 6 } & $M$ & $S E$ & & $M$ & $S E$ \\
\hline Valid & & & & \\
Same & 609 & 18.4 & & 5.3 & 1.11 \\
Different & 629 & 20.3 & & 7.4 & 1.36 \\
Neutral & & & & \\
Same & 616 & 17.7 & & 6.3 & 1.21 \\
Different & 621 & 18.7 & 6.9 & 1.36 \\
\hline
\end{tabular}


nificant object effect. Importantly, a similar effect was not found in the neutral cue block.

It is likely that the pattern of data above reflects a difference in the need for attentional shift between the valid and neutral blocks. In the valid cue block, the cue was $100 \%$ valid. It makes sense for participants to focus attention to the location of the cue prior to its onset, and to shift attention to the target locations as soon as the cue could be interpreted. In the neutral cue block, the cue was uninformative. Participants would have no incentive to attend to the cue. Although the cue was an abrupt onset, which attracts attention under most circumstances (Yantis $\&$ Jonides, 1984), prior research has shown that it does not capture attention in violation of participants' intentions (Yantis \& Jonides, 1990). Furthermore, there is evidence that even when an endogenous cue is informative, the participants can still ignore it when they are instructed to do so (Jonides, 1981). With respect to the present experiment, if the cue in the neutral block could indeed be successfully ignored, there would be no need to shift attention between the cue and the targets.

Several studies have demonstrated that shifting attention between different parts of a stimulus pattern facilitates object-based deployment of attention (Brown \& Denney, 2007; Lamy \& Egeth, 2002; Shomstein \& Yantis, 2002). In several experiments, Lamy and Egeth reported that when targets were presented against background objects so that they belonged to different uniformly connected (UC) regions as in the present Experiments (see Watson $\&$ Kramer, 1999, for the distinction between single UC regions and multiple or grouped UC regions), object-based attention was observed only when a shift of attention was necessary to perform the task. Our finding of the differential degrees of the object effects in the valid and neutral blocks is consistent with the notion that attentional shift facilitates the deployment of object-based attention.

Given the nature of the task, one would assume that the best strategy in the neutral block of Experiment 2 was to adopt a wide attentional focus that included all of the four ends of the stimulus pattern at the beginning of each trial. If that was the case, did the lack of a significant object effect in our results contradict the findings of Goldsmith and Yeari (2003), whose participants demonstrated objectbased attentional distribution when they adopted a wide extent of attentional focus? Among other methodological differences, an important difference between our study and that of Goldsmith and Yeari is the stimulus pattern. There is evidence that whereas the two-bar stimulus configuration used in Goldsmith and Yeari is more likely to be perceived as being made of two separate entities, the cross-like stimulus pattern in the present Experiments is more likely to be seen as being made of a single object (see note 2 of Chen \& Cave, 2006). Since the quality of object representations is known to influence the manifestation of the object effect (Avrahami, 1999), it is perhaps not surprising that the participants in the two studies differed in the manifestation of the object effect.

In light of the differential patterns of data in the valid and neutral blocks, it is unlikely that the cue in Experi- ment 1 served only as a sensory cue. Instead, the results of Experiment 2 suggest that the participants interpreted the cue when it was informative, and that they used it to guide attention.

\section{EXPERIMENT 3}

In Experiments 1 and 2, the stimulus pattern was displayed for about a second before the onset of the cue. This was to provide the participants with sufficient time to process its structure. In Experiment 3, we decreased the exposure duration. If the key to object-based attention is the quality of object representations, and if it takes time to establish object representations, shortening the exposure duration of the stimulus pattern should impair the quality of object representations, thereby eliminating the object effect observed in the previous experiments.

\section{Method}

All aspects of the method in Experiment 3 were identical to those in Experiment 1, with the exception that instead of 1,005 msec, the stimulus pattern was presented for $120 \mathrm{msec}$. Fourteen new participants took part in the experiment.

\section{Results and Discussion}

The data are shown in Table 3. As predicted, there was no significant difference between the same-object condition $(M=579 \mathrm{msec}$, with $7.1 \%$ error $)$ and the differentobject condition ( $M=583 \mathrm{msec}$, with $7.3 \%$ error $)$ in either RT $[t(13)=1.69$, n.s. $]$ or accuracy $[t(13)=0.24$, n.s. $]$.

To confirm that the magnitudes of the object effects were different in Experiments 1 and 3, we performed a mixed ANOVA on RTs with object condition as a withinsubjects variable and experiment as a between-subjects variable. The results showed a significant main effect of object $[F(1,26)=18.26, p<.001]$, with longer RTs in the different-object condition $(M=611 \mathrm{msec})$ than in the same-object condition $(M=602 \mathrm{msec})$. More important, there was also a significant object $\times$ experiment interaction $[F(1,26)=5.92, p<.05]$, indicating a larger object effect in Experiment $1(14 \mathrm{msec})$ than in Experiment 3 $(4 \mathrm{msec})$. The overall response latencies in the two Experiments did not differ significantly $[F(1,26)=2.95$, n.s.]. Because the only methodological difference between the two Experiments was the presentation duration of the stimulus pattern, it seems reasonable to attribute the elimination of the object effect in Experiment 3 to the shortened exposure time of the stimulus pattern.

One might ask whether the results of Experiment 3 are entirely consistent with the results of Law and Abrams (2002), who obtained object effects with an exposure dura-

Table 3

Mean Reaction Times (RTs, in Milliseconds) and Error Rates (Percentage Incorrect), With Standard Errors, for Experiment 3

\begin{tabular}{cccccc}
\hline & \multicolumn{2}{c}{ RT } & & \multicolumn{2}{c}{ \% Error } \\
\cline { 2 - 3 } \cline { 5 - 6 } & $M$ & $S E$ & & $M$ & $S E$ \\
\hline Same & 579 & 13.8 & & 7.1 & 1.75 \\
Different & 583 & 13.3 & & 7.3 & 1.44 \\
\hline
\end{tabular}


tion of $186 \mathrm{msec}$. In our experiment, because the stimulus pattern remained on the screen throughout a trial, its actual exposure duration was $360 \mathrm{msec}$ if we include the presentation durations of the cue and the target display. Whereas Law and Abrams employed an exogenous cue, we used an endogenous cue, and the rising time of attention triggered by an exogenous cue is faster than that triggered by an endogenous cue (Jonides, 1981; Müller \& Rabbitt, 1989; Nakayama \& Mackeben, 1989). Müller and Rabbitt used a spatial cuing paradigm in which the task was to respond to a target after either a peripheral/exogenous cue or a central/endogenous cue. The two types of cue were shown in separate blocks, and the cue-target stimulus onset asynchrony (SOA) ranged from 100 to $725 \mathrm{msec}$. The peak performance on valid trials was at an SOA of $175 \mathrm{msec}$ when the peripheral cue was used; it was at an SOA of $400 \mathrm{msec}$ when the central cue was employed. These results suggest that exogenous and endogenous cues follow different time courses in their guidance of attention. Because the present experiment used endogenous cues, longer exposure duration was needed for object-based attention to manifest itself than the typical exposure duration required with presentation of exogenous cues. Thus, given the difference in rising time between the two types of cue, the difference between timing in the present experiment and that in Law and Abrams is to be expected.

The differential magnitude of the object effect in Experiments 1 and 3 suggests that the deployment of object-based attention requires the encoding of the structural organization of a stimulus pattern, which in turn takes time to develop. When a stimulus is flashed only briefly, as in Experiment 3, participants are unlikely to have enough time to finish the processing of its structural organization before the representation of the target emerges. As a result, no object effect is observed. A similar interpretation has been offered by Law and Abrams (2002), who also attributed the absence of the object effect in one of their Experiments to insufficient processing time for segmenting the stimulus into different objects. As for the exact exposure duration required for the deployment of attention, that may depend on a number of factors, including, but not limited to, the nature of the task (Duncan, 1984; Kramer \& Watson, 1995), the perceptual organization of the stimulus pattern (Chen, 1998; Chen \& Cave, 2006), the number of distractors to be filtered out (Baylis \& Driver, 1993; Chen, 2000), and the extent of attentional focus (Goldsmith \& Yeari, 2003).

\section{EXPERIMENT 4}

Experiment 4 was designed to provide converging evidence that the valid cue used in our Experiments functioned as an endogenous cue rather than as an exogenous one. So far, the cue had always appeared on the surface of the large rectangle. Although the results of Experiment 2 made it unlikely that the cue was only sensory in nature and that the object effects observed in the previous Experiments were due to the cue's being on the large rectangle, it was desirable to obtain converging evidence against the hypothesis that the cue in Experiment 1 acted as an exogenous cue.

To minimize the possibility of exogenous cuing, we changed the stimulus displays in two ways. First, instead of onset, we used offset to indicate the appearance of the cue (see Figure 2). We achieved this by displaying a pair of two-headed arrows at the beginning of each trial, and then removing one of the arrows to reveal the cue. Because the cue was no longer formed by abrupt onsets, it was less likely that it would function as an exogenous cue. Second, to ensure that the local transients caused by the offset of the arrow were not systematically associated with any specific rectangle, we increased the size of the arrows so that they extended into the surface of the two smaller rectangles. A successful replication of the results of Experiment 1 would provide strong evidence that the object effect observed in Experiment 1 was obtained with an endogenous cue.

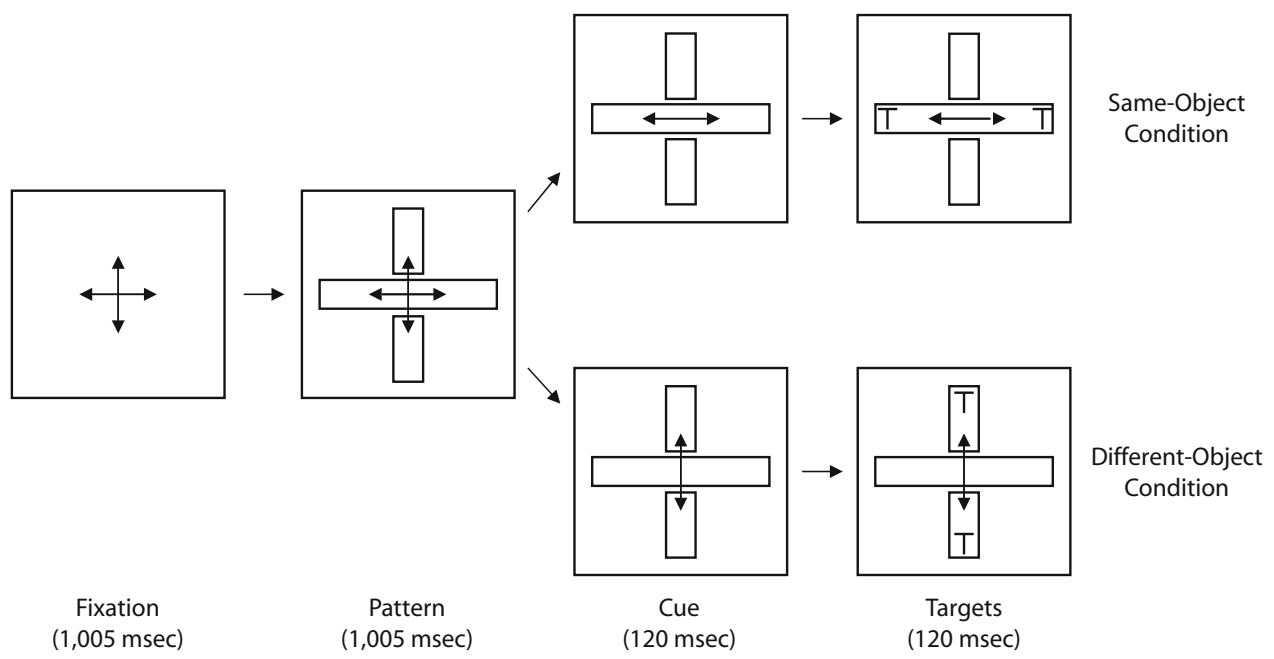

Figure 2. Examples of stimulus displays from Experiment 4. The cue was an offset instead of an onset, and it extended into the surface of the two smaller rectangles. 
Table 4

Mean Reaction Times (RTs, in Milliseconds) and Error Rates (Percentage Incorrect), With Standard Errors, for Experiment 4

\begin{tabular}{lccccc}
\hline & \multicolumn{3}{c}{$\mathrm{RT}$} & & \multicolumn{2}{c}{$\%$ Error } \\
\cline { 2 - 3 } \cline { 5 - 6 } & $M$ & $S E$ & & $M$ & $S E$ \\
\hline Same & 610 & 23.1 & & 6.1 & 1.03 \\
Different & 622 & 24.0 & & 5.7 & 1.09 \\
\hline
\end{tabular}

\section{Method}

The method was identical to that of Experiment 1 except for the following changes. Instead of a cross, the fixation display consisted of a pair of two-headed arrows that subtended $3.25^{\circ}$ of visual angle in both length and width. They stayed on the screen for 1,005 msec and were then joined by the stimulus pattern. As in Experiments 1-3, the stimulus pattern remained on the screen throughout an entire trial After 1,005 $\mathrm{msec}$, one of the arrows would disappear, leaving the other arrow on the screen for another $120 \mathrm{msec}$. Then, two target letters were shown for $120 \mathrm{msec}$ at the locations indicated by the arrow cue. Fourteen new participants volunteered for the experiment.

\section{Results and Discussion}

Table 4 shows the data. Consistent with the findings of Experiment 1, RTs were faster in the same-object condition $(M=610 \mathrm{msec})$ than in the different-object condition $(M=622 \mathrm{msec})[t(13)=3.52, p<.01]$. There was no difference in accuracy between the two conditions $[6.1 \%$ and $5.7 \%$ error rates for the same- and different-object conditions, respectively; $t(13)=0.67$, n.s.].

To verify that there was no qualitative difference in the magnitude of the object effect between Experiments 1 and 4, we performed a mixed ANOVA on RTs. The results indicated a significant main effect of object $[F(1,26)=$ 28.32, $p<.01$ ], with longer RTs in the different-object condition $(M=631 \mathrm{msec})$ than in the same-object condition $(M=618 \mathrm{msec})$. Neither the main effect of experiment $[F(1,26)<1$, n.s. $]$ nor the object $\times$ experiment interaction $[F(1,26)<1$, n.s. $]$ reached statistical significance.

The results of Experiment 4 were remarkably similar to those of Experiment 1. They demonstrate that an object effect can be obtained when the cue is endogenous and when prioritizing items for search is not required.

\section{EXPERIMENT 5}

Experiment 5 was conducted in order to confirm that the differential object effects observed in Experiments 1 and 3 could also be replicated with the large offset cue. The design of the experiment was identical to that of Experiment 4 , with the exception of the shortened presentation duration for the stimulus pattern. Given the results of the

Table 5

Mean Reaction Times (RTs, in Milliseconds) and Error Rates (Percentage Incorrect), With Standard Errors, for Experiment 5

\begin{tabular}{llllll}
\hline & \multicolumn{2}{c}{$\mathrm{RT}$} & & \multicolumn{2}{c}{$\%$ Error } \\
\cline { 2 - 3 } \cline { 5 - 6 } & $M$ & & & $M$ & $S E$ \\
\hline Same & 629 & & & $M$ & \\
Different & 630 & 25.9 & & 4.0 & 0.45 \\
& & &
\end{tabular}

previous experiments, we anticipated no significant difference between the same- and different-object conditions.

\section{Method}

The stimulus pattern was shown for $120 \mathrm{msec}$ in Experiment 5, instead of for $1,005 \mathrm{msec}$. Other than this, all aspects of the experiment were identical to those of Experiment 4. Fourteen new participants from the same pool took part in the study.

\section{Results and Discussion}

The RT and accuracy data are shown in Table 5. No significant differences were found between the sameobject condition ( $M=629 \mathrm{msec}$, with $4.0 \%$ error) and the different-object condition ( $M=630 \mathrm{msec}$, with $3.7 \%$ error $)$ in RT $[t(13)=0.34$, n.s.] or accuracy $[t(13)=0.25$, n.s. $]$.

As with Experiments 1 and 3, a combined analysis was performed on the RT data of Experiments 4 and 5 in order to verify that the magnitude of object effects differed as a function of stimulus duration. The results showed a significant main effect of object $[F(1,26)=6.93, p<$ $.05]$, with longer RTs in the different-object condition $(M=626 \mathrm{msec})$ than in the same-object condition $(M=$ $620 \mathrm{msec}$ ), and a significant object $\times$ experiment interaction $[F(1,26)=4.54, p<.05]$, with the object effect larger in Experiment $4(12 \mathrm{msec})$ than in Experiment 5 $(1 \mathrm{msec})$. There was no significant difference in the main effect of experiment $[F(1,26)<1$, n.s. $]$.

In addition to the analyses above, we also conducted a mixed ANOVA on the RT data of Experiments 3 and 5 in order to ensure that there was no qualitative difference between them. No significant effects were found $[F(1,26)=$ 1.39 , n.s., for object; $F(1,26)=2.87$, n.s., for experiment; or $F(1,26)=0.36$, n.s., for the object $\times$ experiment interaction]. These results confirmed that stimulus duration is an important factor in the establishment of object representations, which in turn is critical in the manifestation of object effects.

\section{GENERAL DISCUSSION}

These Experiments add to the body of evidence demonstrating that object-based attention can appear or disappear depending on specific circumstances, and they provide a clearer outline of the factors that govern objectbased attention.

\section{Sensory Enhancement Versus \\ Search Prioritization}

In the majority of the Experiments reported here, the cues were $100 \%$ valid, so that the target locations were known with certainty before they appeared. The fact that object-based attention arose in these circumstances demonstrates that it is widely used, even when search is unnecessary. These results provide converging evidence for our previous finding that object-based attention arises due to sensory enhancement of a selected item or its region (Chen \& Cave, 2006), as well as another piece of evidence against a search prioritization explanation of object-based attention (Shomstein \& Yantis, 2002). 


\section{Endogenous Versus Exogenous Cuing}

The cues in the present Experiments were endogenous, yet attention was affected by the object boundaries. Thus, although object-based attention is often associated with exogenous cuing, as has been noted by Macquistan (1997) and other researchers (Arrington \& Dagenbach, 2000; Neely \& Dagenbach, 1996), our findings provide another demonstration that it can also occur with endogenous cuing. These results go beyond those of Goldsmith and Yeari (2003) by showing that object-based attention can arise in a cuing task that differs from theirs in a number of ways, including the $100 \%$ certainty about the target location that removes any aspect of search from the task.

\section{Attentional Shift}

The differential magnitudes of the object effects in the valid and neutral blocks of Experiment 2 are consistent with the notion that attentional shift between different parts of a stimulus pattern facilitates object-based deployment of attention (Brown \& Denney, 2007; Lamy \& Egeth, 2002; Shomstein \& Yantis, 2002). Whereas a significant object effect was found when participants had the incentive to shift attention from the cue to the target locations, no object effect was observed when such incentive was eliminated by the use of an uninformative neutral cue instead of a valid one.

Why does attentional shift facilitate object-based attention? Goldsmith and Yeari (2003) proposed that the shifting of attention encourages participants to spread attention widely over the stimulus pattern, which in turn enhances the creation and maintenance of object-based representations. The key to object-based deployment of attention is therefore the quality of object-based representations, a notion consistent with the proposal of Avrahami (1999). It is worth noting that although both attentional shift and spread of attention are conducive to object-based attention, neither of them is a necessary condition. In a previous article (Chen \& Cave, 2006), we showed that as long as a stimulus pattern is subjectively parsed as comprising multiple objects, object-based attention can be observed even when there is no need to shift attention. Similarly, in the neutral block of Experiment 2 reported here, although the best strategy was to adopt a wide attentional focus that covered the entire stimulus pattern, there was no evidence of object-based attention, presumably because robust object representations could not be established and/or maintained due to the nature of the stimulus pattern used in the experiment. We share Goldsmith and Yeari's view that having viable object representations is a critical factor of deploying object-based attention.

\section{Object-Based Attention Takes Time to Develop}

Experiments 3 and 5 showed that object-based attention can be prevented by shortening the exposure time of the object stimuli. Display timing has been a factor in other studies of object-based attention. Lavie and Driver (1996) found object effects in their first three Experiments but not in their fourth. They attributed the difference to the introduction of a spatial cue in the fourth experiment, and their explanation that the object effect disappears with the narrower focus of attention is consistent with the results of other studies noted above. However, the exposure time for the two-object display was shortened from 177 to $130 \mathrm{msec}$ in their fourth experiment. Law and Abrams (2002) performed a similar experiment with exposure times of 129 and $186 \mathrm{msec}$, and demonstrated that shortening the exposure time in this way was sufficient to eliminate the object effect. Thus, the results from Experiments 3 and 5 are generally consistent with Law and Abrams's conclusion that object-based attention can be prevented with short exposure times.

However, it is interesting that exposure times of 177 or $186 \mathrm{msec}$ were sufficient for object-based attention in these earlier studies, whereas our Experiment 3 showed no object-based attention when the three rectangles were displayed for a total of $240 \mathrm{msec}$ before the letters appeared. As noted earlier, the extra time may have been necessary in our experiment in order to allow for the interpretation of the endogenous cue. Extra time may also have been required in order to parse and organize the three rectangles in our experiments, as opposed to the two lines used by Lavie and Driver (1996) and by Law and Abrams (2002). Our task also required a more complex allocation of attention, with two cued locations on opposite sides of the display, which may also have required more preparation time.

\section{The Role of Object-Based Attention in Visual Processing and the Generation of Responses}

These results, taken together and combined with previous results, show the factors governing object-based attention to be fairly complex. Note that participants are able to proceed with this visual comparison task with or without the object representations. Visual processing apparently does not wait for the object representations to be completed, at least not in such a way as to influence performance. This pattern suggests that the object-based system is available to help in directing the allocation of spatial attention but is not necessarily an integral part of the attention process. The fact that we can direct attention spatially with no objects in the visual field is additional evidence for the flexibility of the attention system.

These results also suggest that there is substantial flexibility in how fully a stimulus is segregated into objects. In some tasks (Chen \& Cave, 2006, Experiment 1; and the neutral block of Experiment 2 in the present study; Shomstein \& Yantis, 2002), the objects are apparently not encoded or not fully encoded, and thus they do not affect attention. Interestingly, it does not take much to trigger object segregation. The object boundaries do not have to be relevant to the task in order to be encoded. Inducing participants to shift attention or prior experience with the objects (Chen \& Cave, 2006, Experiment 2) was enough to trigger object segregation. Thus, object segregation must occur easily enough so that it is often triggered spontaneously, even though it is not an automatic processing stage.

Although object-based attention may not require much effort, these Experiments show that it does require a cer- 
tain amount of time. In some tasks, a response is needed too quickly to wait for object segregation to occur. Luckily, in those cases the visual system can allocate attention and generate a response without it.

\section{AUTHOR NOTE}

We thank Morris Goldsmith, Kate Arrington, and an anonymous reviewer for their helpful comments. Correspondence concerning this article should be addressed to Z. Chen, Department of Psychology, University of Canterbury, Private Bag 4800, Christchurch, New Zealand (e-mail: zhe.chen@canterbury.ac.nz).

\section{REFERENCES}

Abrams, R. A., \& Law, M. B. (2000). Object-based visual attention with endogenous orienting. Perception \& Psychophysics, 62, 818-833.

Arrington, C. M., \& Dagenbach, D. (2000, November). The reliability of object-based attention following peripheral and central cues. Poster presented at the 41st Annual Meeting of the Psychonomic Society, New Orleans.

Avrahami, J. (1999). Objects of attention, objects of perception. Perception \& Psychophysics, 61, 1604-1612.

Baylis, G. C., \& Driver, J. (1993). Visual attention and objects: Evidence for hierarchical coding of location. Journal of Experimental Psychology: Human Perception \& Performance, 19, 451-470.

Brown, J. M., \& Denney, H. I. (2007). Shifting attention into and out of objects: Evaluating the processes underlying the object advantage. Perception \& Psychophysics, 69, 606-618.

CHEN, Z. (1998). Switching attention within and between objects: The role of subjective organization. Canadian Journal of Experimental Psychology, 52, 7-16.

Chen, Z. (2000). An object-based cost of visual filtering. Perception \& Psychophysics, 62, 482-495.

Chen, Z., \& CAVE, K. R. (2006). Reinstating object-based attention under positional certainty: The importance of subjective parsing. Perception \& Psychophysics, 68, 992-1003.

Desimone, R., \& Duncan, J. (1995). Neural mechanisms of selective visual attention. Annual Review of Neuroscience, 18, 193-222.

Duncan, J. (1984). Selective attention and the organization of visual information. Journal of Experimental Psychology: General, 113, 501-517.

Egly, R., Driver, J., \& Rafal, R. D. (1994). Shifting visual attention between objects and locations: Evidence from normal and parietal lesion subjects. Journal of Experimental Psychology: General, 123, 161-177.

Goldsmith, M., \& Yeari, M. (2003). Modulation of object-based attention by spatial focus under endogenous and exogenous orienting. Journal of Experimental Psychology: Human Perception \& Performance, 29, 897-918.

Harms, L., \& Bundesen, C. (1983). Color segregation and selective attention in a nonsearch task. Perception \& Psychophysics, 33, 11-19.

HunT, S. M. J. (1994). MacProbe: A Macintosh-based experimenter's workstation for the cognitive sciences. Behavior Research Methods, Instruments, \& Computers, 26, 345-351.

Jonides, J. (1981). Voluntary vs. automatic control over the mind's eye's movement. In J. B. Long \& A. D. Baddeley (Eds.), Attention and performance IX (pp. 187-203). Hillsdale, NJ: Erlbaum.

KIM, M.-S., \& CAVE, K. R. (2001). Perceptual grouping via spatial selection in a focused-attention task. Vision Research, 41, 611-624.

Kramer, A. F., \& Jacobson, A. (1991). Perceptual organization and focused attention: The role of objects and proximity in visual processing. Perception \& Psychophysics, 50, 267-284.

Kramer, A. F., \& Watson, S. E. (1995). Object-based visual selection and the principle of uniform connectedness. In A. F. Kramer, M. G. H. Coles, \& G. D. Logan (Eds.), Converging operations in the study of visual selective attention (pp. 395-414). Washington, DC: American Psychological Association.

LAMy, D., \& EgETH, H. (2002). Object-based selection: The role of attentional shifts. Perception \& Psychophysics, 64, 52-66.

Lavie, N., \& Driver, J. (1996). On the spatial extent of attention in object-based visual selection. Perception \& Psychophysics, 58, 1238-1251.

LaW, M. B., \& Abrams, R. A. (2002). Object-based selection within and beyond the focus of spatial attention. Perception \& Psychophysics, 64, 1017-1027.

MacQuistan, A. (1997). Object-based allocation of visual attention in response to exogenous, but not endogenous, spatial precues. Psychonomic Bulletin \& Review, 4, 512-515.

Moore, C. M., Yantis, S., \& Vaughan, B. (1998). Object-based visual selection: Evidence from perceptual completion. Psychological Science, 9, 104-110.

MülleR, H. J., \& RabbitT, P. M. A. (1989). Reflexive and voluntary orienting of visual attention: Time course of activation and resistance to interruption. Journal of Experimental Psychology: Human Perception \& Performance, 15, 315-330.

Nakayama, K., \& Mackeben, M. (1989). Sustained and transient components of focal visual attention. Vision Research, 29, 1631-1647.

Neely, C. A., \& Dagenbach, D. (1996, November). Exogenous and endogenous cuing: Spatial versus object-based visual attention. Poster presented at the 37th Annual Meeting of the Psychonomic Society, Chicago.

Shomstein, S., \& Yantis, S. (2002). Object-based attention: Sensory modulation or priority setting? Perception \& Psychophysics, 64, 41-51.

Watson, S. E., \& Kramer, A. F. (1999). Object-based visual selective attention and perceptual organization. Perception \& Psychophysics, 61, 31-49.

Yantis, S., \& Jonides, J. (1984). Abrupt visual onsets and selective attention: Evidence from visual search. Journal of Experimental Psychology: Human Perception \& Performance, 10, 601-621.

YANTIS, S., \& Jonides, J. (1990). Abrupt visual onsets and selective attention: Voluntary versus automatic allocation. Journal of Experimental Psychology: Human Perception \& Performance, 16, 121-134.

\section{NOTE}

1. We thank Morris Goldsmith and an anonymous reviewer for suggesting this.

(Manuscript received November 5, 2007; revision accepted for publication May 7, 2008.) 